\title{
Energy conditions for a generally coupled scalar field outside a reflecting sphere
}

\author{
Delia Schwartz-Perlov* and Ken D. Olum \\ Institute of Cosmology, Department of Physics and Astronomy, \\ Tufts University, Medford, MA 02155
}

\begin{abstract}
We analyze the stress-energy tensor, and the resulting energy conditions, for a scalar field with general curvature coupling, outside a perfectly reflecting sphere with Dirichlet boundary conditions. For conformal coupling we find that the null energy condition is always obeyed, and therefore the averaged null energy condition (ANEC) is also obeyed. Since the ANEC is independent of curvature coupling, we conclude that the ANEC is obeyed for scalar fields with any curvature coupling in this situation. We also show explicitly how the spherical case goes over to that of a flat plate as one approaches the sphere.
\end{abstract}

PACS numbers: $03.65 . \mathrm{Nk}$ 04.20.Gz

*Electronic address: Delia.Perlov@tufts.edu

${ }^{\dagger}$ Electronic address: kdo@cosmos.phy.tufts.edu 


\section{INTRODUCTION}

From our everyday experience we expect the energy density of any substance to be a positive quantity. However, quantum field theory allows the existence of states which have negative energy densities, as first shown theoretically by Hendrik Casimir in 1948 [1]. Casimir found that the energy density between two neutral conducting plates was negative and resulted in an attractive force between the plates. This attractive "Casimir" force has only recently been accurately measured [2].

The reality of negative energy densities has opened the possibility of exotic physical situations in general relativity, including wormholes and time travel. With no restriction on the stress-energy tensor, one can simply write down any desired space-time geometry and solve Einstein's equations in reverse to find the matter stress-energy that will produce that geometry. To prevent exotic phenomena requires energy conditions that all matter must obey.

The simplest of these is the weak energy condition (WEC), which states that no observer sees negative energy density,

$$
T_{\mu \nu} V^{\mu} V^{\nu} \geq 0
$$

where $T_{\mu \nu}$ is the stress-energy tensor and $V^{\mu}$ is any timelike vector. If instead we consider null $V^{\mu}$ in Eq. (11), we get the null energy condition (NEC). If the WEC is obeyed at some location for all $V$, then by continuity the NEC must also be obeyed. These conditions, however, are violated by the Casimir effect between parallel plates.

The averaged weak energy condition (AWEC) allows the WEC to be violated locally, but holds that when one integrates the WEC over a complete geodesic with tangent vector $V^{\mu}$, the result must be non-negative,

$$
\int_{-\infty}^{\infty} d x T_{\mu \nu} V^{\mu} V^{\nu} \geq 0 .
$$

When $V^{\mu}$ is null, Eq. (2) represents the averaged null energy condition (ANEC). The AWEC is also violated by the Casimir effect, but the ANEC is obeyed, because the geodesic cannot pass through the plates (considered as ideal boundaries) and so must run parallel to them, in which case all contributions cancel.

The ANEC is the most important condition for our purposes, since its validity is sufficient to rule out many exotic phenomena, such as traversable wormholes [3], superluminal travel [4], and closed timelike curves [5], and to prove singularity theorems [6, 7, 8] $]^{1}$. No ANEC violations are known for a scalar field in flat space quantum field theory. In [9], we computed the stress-energy tensor outside a Dirichlet sphere and found that ANEC was always obeyed. Here, we extend this work to arbitrary curvature coupling.

The stress energy tensor for an arbitrarily coupled scalar field outside a sphere was found by Saharan [10]. We reproduce these results using a similar technique, based on the calculational framework developed in [11]. In Secs. [I] and III we obtain a general expression for the vacuum stress-energy tensor outside a spherically symmetric scattering center. In Sec. IV we specialize to perfectly reflecting boundary conditions on a sphere. In Sec. V] we show how our results give analytic expressions for the stress-energy tensor in the large $r$ limit.

\footnotetext{
${ }^{1}$ For superluminal travel, we integrate only over the path to be traveled, and for singularity theorems only over the part of the geodesic to the future of a trapped surface.
} 
In Sec. VI we calculate the NEC numerically and find that it is always obeyed for conformal coupling. Since the NEC is obeyed for conformal coupling at every point outside the sphere, we know that the ANEC is obeyed too. Furthermore, the ANEC is coupling constant independent, so we can conclude that the ANEC is obeyed for all scalar field couplings.

In Sec. VII we show how our results approach the usual stress-energy tensor for a flat Dirichlet plate for points very close to the sphere. We follow with conclusions in Sec. VIII,

\section{STRESS-ENERGY TENSOR FOR GENERAL COUPLING}

The stress-energy tensor for a massless real scalar field is

$$
T_{\mu \nu}=\partial_{\mu} \phi \partial_{\nu} \phi-\frac{1}{2} \eta_{\mu \nu} \partial^{\lambda} \phi \partial_{\lambda} \phi+\xi\left[\eta_{\mu \nu} \partial_{\lambda} \partial^{\lambda}\left(\phi^{2}\right)-\partial_{\mu} \partial_{\nu}\left(\phi^{2}\right)\right],
$$

where $\xi$ is the curvature coupling, and we are working in the limit where $|\phi| \ll m_{\text {Planck }}$. For conformal coupling in $3+1$ dimensions, $\xi=1 / 6$.

If we consider a static, spherically symmetric system, the stress tensor can be written in terms of the energy density $\rho$, the radial pressure $p_{r}$ and the tangential pressure $p_{\perp}$. For example, on the $x$ axis we have $T_{00}=\rho, T_{x x}=p_{r}, T_{y y}=T_{z z}=p_{\perp}$, with other components vanishing.

From Eq. (3), these components are

$$
\begin{aligned}
\rho & =\frac{1}{2}\left[\dot{\phi}^{2}+\left(\partial_{r} \phi\right)^{2}+2\left(\partial_{\perp} \phi\right)^{2}\right]-\xi\left[\partial_{r}^{2}\left(\phi^{2}\right)+2 \partial_{\perp}^{2}\left(\phi^{2}\right)\right] \\
p_{r} & =\frac{1}{2}\left[\dot{\phi}^{2}+\left(\partial_{r} \phi\right)^{2}-2\left(\partial_{\perp} \phi\right)^{2}\right]+\xi\left[2 \partial_{\perp}^{2}\left(\phi^{2}\right)-\partial_{0}^{2}\left(\phi^{2}\right)\right] \\
p_{\perp} & =\frac{1}{2}\left[\dot{\phi}^{2}-\left(\partial_{r} \phi\right)^{2}\right]+\xi\left[\partial_{\perp}^{2}\left(\phi^{2}\right)+\partial_{r}^{2}\left(\phi^{2}\right)-\partial_{0}^{2}\left(\phi^{2}\right)\right]
\end{aligned}
$$

where $\partial_{\perp}$ denotes the derivative along a tangential line. (By rotational symmetry the choice of line will not matter.) The vacuum expectation value $\left\langle\phi^{2}\right\rangle$ depends only on $r$, since the system is static and rotationally symmetric. Thus $\left\langle\partial_{0}^{2}\left(\phi^{2}\right)\right\rangle=\partial_{0}^{2}\left\langle\phi^{2}\right\rangle=0$. The second

derivative in the tangential direction does not vanish, however. There is still a term $\partial_{\perp}^{2}\left\langle\phi^{2}\right\rangle=$ $(1 / r) \partial_{r}\left\langle\phi^{2}\right\rangle$. Thus

$$
\begin{aligned}
\rho & =\frac{1}{2}\left[\dot{\phi}^{2}+\left(\partial_{r} \phi\right)^{2}+2\left(\partial_{\perp} \phi\right)^{2}\right]-\xi\left[\partial_{r}^{2}\left(\phi^{2}\right)+\frac{2}{r} \partial_{r}\left(\phi^{2}\right)\right] \\
p_{r} & =\frac{1}{2}\left[\dot{\phi}^{2}+\left(\partial_{r} \phi\right)^{2}-2\left(\partial_{\perp} \phi\right)^{2}\right]+\frac{2 \xi}{r} \partial_{r}\left(\phi^{2}\right) \\
p_{\perp} & =\frac{1}{2}\left[\dot{\phi}^{2}-\left(\partial_{r} \phi\right)^{2}\right]+\xi\left[\frac{1}{r} \partial_{r}\left(\phi^{2}\right)+\partial_{r}^{2}\left(\phi^{2}\right)\right]
\end{aligned}
$$

If we are interested only in the null energy condition, then the terms proportional to the metric do not contribute, and we have

$$
T_{\mu \nu} V^{\mu} V^{\nu}=\left(V^{\alpha} \partial_{\alpha} \phi\right)^{2}-\xi V^{\mu} V^{\nu} \partial_{\mu} \partial_{\nu}\left(\phi^{2}\right) .
$$

If $V=(1, \mathbf{v})$ with $|\mathbf{v}|=1$, then

$$
T_{\mu \nu} V^{\mu} V^{\nu}=\dot{\phi}^{2}+v_{r}^{2}\left(\partial_{r} \phi\right)^{2}+v_{\perp}^{2}\left(\partial_{\perp} \phi\right)^{2}-\xi\left[v_{r}^{2} \partial_{r}^{2}\left(\phi^{2}\right)+\frac{v_{\perp}^{2}}{r} \partial_{r}\left(\phi^{2}\right)\right]
$$

where $v_{\perp}$ is the magnitude of the velocity in the tangential direction, $v_{\perp}^{2}+v_{r}^{2}=1$. 


\section{VACUUM EXPECTATION VALUES}

In order to calculate the vacuum expectation value of the stress-energy tensor, it is sufficient to compute the expectation values of $\phi^{2}, \dot{\phi}^{2},\left(\partial_{r} \phi\right)^{2}$, and $\left(\partial_{\perp} \phi\right)^{2}$.

We can write the quantum field $\phi$ in spherical modes

$$
\phi(r, \Omega, t)=\sum_{\ell=0}^{\infty} \sum_{m=-\ell}^{\ell} \int_{0}^{\infty} d k \frac{k}{\sqrt{\pi \omega}} \psi_{k}^{\ell}(r)^{*} Y_{\ell m}(\Omega)^{*} e^{i \omega t} a_{k \ell m}^{\dagger}+\text { c.c. }
$$

where $\omega=k$ since the field is massless.

The wavefunctions $\psi_{k}^{\ell}(r)$ satisfy the time-independent radial Schrödinger equation

$$
\left(-\frac{d^{2}}{d r^{2}}-\frac{2}{r} \frac{d}{d r}+\frac{\ell(\ell+1)}{r^{2}}\right) \psi_{k}^{\ell}(r)=k^{2} \psi_{k}^{\ell}(r) .
$$

normalized

$$
\int_{0}^{\infty} k^{2} r^{2} \psi_{k}^{\ell}(r)^{*} \psi_{k^{\prime}}^{\ell}(r) d r=\frac{\pi}{2} \delta\left(k-k^{\prime}\right)
$$

so that the free wave function is just $\psi_{k}^{\ell}(r)=j_{\ell}(k r)$ where $j$ is the spherical Bessel function $j_{\ell}(z)=\sqrt{\pi /(2 z)} J_{\ell+1 / 2}(z)$.

From Eq. (12) we can compute the vacuum expectation value of $\phi^{2}$. There will be no dependence on the angular position, so we can sum over the spherical harmonics using

$$
\sum_{m=-\ell}^{\ell}\left|Y_{\ell m}(\Omega)\right|^{2}=\frac{2 \ell+1}{4 \pi}
$$

to get

$$
\left\langle\phi^{2}\right\rangle=\sum_{\ell} \frac{2 \ell+1}{4 \pi^{2}} \int_{0}^{\infty} d k \frac{k^{2}}{\omega}\left(\left|\psi_{k}^{\ell}(r)\right|^{2}-j_{\ell}(k r)^{2}\right)
$$

where we have subtracted the free wavefunctions. No other counterterms are necessary, because we are considering locations in empty space without any potential.

The vacuum expectation values for the time and radial derivatives are straightforward,

$$
\begin{aligned}
\left\langle\dot{\phi}^{2}\right\rangle & =\sum_{\ell} \frac{2 \ell+1}{4 \pi^{2}} \int_{0}^{\infty} d k k^{2} \omega\left(\left|\psi_{k}^{\ell}(r)\right|^{2}-j_{\ell}(k r)^{2}\right), \\
\left\langle\left(\partial_{r} \phi\right)^{2}\right\rangle & =\sum_{\ell} \frac{2 \ell+1}{4 \pi^{2}} \int_{0}^{\infty} d k \frac{k^{2}}{\omega}\left(\left|\partial_{r} \psi_{k}^{\ell}(r)\right|^{2}-\left(\partial_{r} j_{\ell}(k r)\right)^{2}\right) .
\end{aligned}
$$

To compute the azimuthal term we need the derivative of the spherical harmonics. Without loss of generality we can work in the equatorial plane where we can take $\partial_{\perp} Y_{\ell m}(\pi / 2, \phi)=$ $(1 / r) \partial_{\phi} Y_{\ell m}(\pi / 2, \phi)=(i m / r) Y_{\ell m}(\pi / 2, \phi)$. Using the formula

$$
\sum_{m=-\ell}^{\ell} m^{2}\left|Y_{\ell m}(\pi / 2, \phi)\right|^{2}=\frac{\ell(\ell+1)(2 \ell+1)}{8 \pi}
$$


we get

$$
\left\langle\left(\partial_{\perp} \phi\right)^{2}\right\rangle=\sum_{\ell} \frac{\ell(\ell+1)(2 \ell+1)}{8 \pi^{2} r^{2}} \int_{0}^{\infty} d k \frac{k^{2}}{\omega}\left(\left|\psi_{k}^{\ell}(r)\right|^{2}-j_{\ell}(k r)^{2}\right) .
$$

Outside any spherically symmetric potential, the wave functions are given by

$$
\psi_{k}^{\ell}(r)=\frac{1}{2}\left[e^{2 i \delta_{\ell}} h_{\ell}^{(1)}(k r)+h_{\ell}^{(2)}(k r)\right]
$$

where $\delta_{\ell}$ is the scattering phase shift in the quantum mechanical problem with the same potential, and the spherical Hankel functions are $h_{\ell}^{(1,2)}(z)=\sqrt{\pi /(2 z)} H_{\ell+1 / 2}^{(1,2)}(z)$. Thus

$$
\left|\psi_{k}^{\ell}(r)\right|^{2}-j_{\ell}(k r)^{2}=\frac{1}{4}\left[\left(e^{2 i \delta_{\ell}}-1\right) h_{\nu}^{(1)}(k r)^{2}+\left(e^{-2 i \delta_{\ell}}-1\right) h_{\nu}^{(2)}(k r)^{2}\right] .
$$

From the appendix of [9] it is easy to show that when we use Eq. (22) in Eqs. (16-1820) we can drop the second term of Eq. (22) and extend the range of integration over $k$ to $-\infty$, with the understanding that $k$ is to be taken above any branch cut on the negative real axis.

Then, following the methods used in [11] and [9] (a similar procedure was used previously in 10]), we convert each expression to a contour integral which we close in the upper half plane. The only contribution to the integral comes from the branch cut along the imaginary $k$ axis. To the right $\omega=\sqrt{k^{2}}=k$, but to the left $\omega=-k$. We then use $h_{\ell}^{(1)}(i x)=-(2 / \pi) i^{-\ell} k_{\ell}(x)$, where $k_{\ell}$ is the modified spherical Bessel function, $k_{\ell}(z)=\sqrt{\pi /(2 z)} K_{\ell+1 / 2}(z)$. With $k=i \kappa$, we obtain

$$
\begin{aligned}
\left\langle\phi^{2}\right\rangle & =-\sum_{\ell}(-)^{\ell} \frac{2 \ell+1}{2 \pi^{4}} \int_{0}^{\infty} d \kappa \kappa\left(e^{2 i \delta_{\ell}}-1\right) k_{\ell}(\kappa r)^{2} \\
\left\langle\dot{\phi}^{2}\right\rangle & =\sum_{\ell}(-)^{\ell} \frac{2 \ell+1}{2 \pi^{4}} \int_{0}^{\infty} d \kappa \kappa^{3}\left(e^{2 i \delta_{\ell}}-1\right) k_{\ell}(\kappa r)^{2} \\
\left\langle\left(\partial_{r} \phi\right)^{2}\right\rangle & =-\sum_{\ell}(-)^{\ell} \frac{2 \ell+1}{2 \pi^{4}} \int_{0}^{\infty} d \kappa \kappa^{3}\left(e^{2 i \delta_{\ell}}-1\right) k_{\ell}^{\prime}(\kappa r)^{2} \\
\left\langle\left(\partial_{\perp} \phi\right)^{2}\right\rangle & =-\sum_{\ell}(-)^{\ell} \frac{\ell(\ell+1)(2 \ell+1)}{4 \pi^{4} r^{2}} \int_{0}^{\infty} d \kappa \kappa\left(e^{2 i \delta_{\ell}}-1\right) k_{\ell}(\kappa r)^{2} .
\end{aligned}
$$

\section{PERFECTLY REFLECTING BOUNDARY CONDITIONS}

For a hard sphere of radius $a$, with Dirichlet boundary conditions,

$$
e^{2 i \delta_{\ell}}=-\frac{h_{\ell}^{(2)}(k a)}{h_{\ell}^{(1)}(k a)}
$$

so that $\phi(a)=0$. Thus

$$
e^{2 i \delta_{\ell}}-1=-\frac{2 j_{\ell}(k a)}{h_{\ell}^{(1)}(k a)}
$$

and

$$
e^{2 i \delta_{\ell}(i \kappa)}-1=(-)^{\ell} \pi \frac{i_{\ell}(\kappa a)}{k_{\ell}(\kappa a)}
$$


since $j_{\ell}(i x)=i^{\ell} i_{\ell}(x)$ where $i_{\ell}(z)=\sqrt{\pi /(2 z)} I_{\ell+1 / 2}(z)$.

Thus

$$
\begin{aligned}
\left\langle\phi^{2}\right\rangle & =-\sum_{\ell} \frac{2 \ell+1}{2 \pi^{3}} \int_{0}^{\infty} d \kappa \kappa \frac{i_{\ell}(\kappa a)}{k_{\ell}(\kappa a)} k_{\ell}(\kappa r)^{2} \\
\left\langle\dot{\phi}^{2}\right\rangle & =\sum_{\ell} \frac{2 \ell+1}{2 \pi^{3}} \int_{0}^{\infty} d \kappa \kappa^{3} \frac{i_{\ell}(\kappa a)}{k_{\ell}(\kappa a)} k_{\ell}(\kappa r)^{2} \\
\left\langle\left(\partial_{r} \phi\right)^{2}\right\rangle & =-\sum_{\ell} \frac{2 \ell+1}{2 \pi^{3}} \int_{0}^{\infty} d \kappa \kappa^{3} \frac{i_{\ell}(\kappa a)}{k_{\ell}(\kappa a)} k_{\ell}^{\prime}(\kappa r)^{2} \\
\left\langle\left(\partial_{\perp} \phi\right)^{2}\right\rangle & =-\sum_{\ell} \frac{\ell(\ell+1)(2 \ell+1)}{4 \pi^{3} r^{2}} \int_{0}^{\infty} d \kappa \kappa \frac{i_{\ell}(\kappa a)}{k_{\ell}(\kappa a)} k_{\ell}(\kappa r)^{2} .
\end{aligned}
$$

in agreement with [10].

From these equations we can compute any combination of stress-energy tensor components. For $V$ null, from Eq. (11),

$$
\begin{aligned}
T_{\mu \nu} V^{\mu} V^{\nu}=\sum_{\ell} \frac{2 \ell+1}{2 \pi^{3}} \int_{0}^{\infty} d \kappa \kappa^{3} \frac{i_{\ell}(\kappa a)}{k_{\ell}(\kappa a)} & \left\{k_{\ell}(\kappa r)^{2}-v_{r}^{2} k_{\ell}^{\prime}(\kappa r)^{2}-\frac{\ell(\ell+1)}{2 \kappa^{2} r^{2}} v_{\perp}^{2} k_{\ell}(\kappa r)^{2}(34)\right. \\
& \left.+\xi\left[\frac{v_{r}^{2}}{\kappa^{2}} \partial_{r}^{2}\left(k_{\ell}(\kappa r)^{2}\right)+\frac{v_{\perp}^{2}}{\kappa^{2} r} \partial_{r}\left(k_{\ell}(\kappa r)^{2}\right)\right]\right\}
\end{aligned}
$$

\section{LARGE DISTANCE BEHAVIOR}

Far away from the sphere, in the large $r$ regime, the stress-energy tensor is dominated by

the $\ell=0$ partial wave contribution. For this case the integrals in Eqs. (30]33) can be done analytically. We find

$$
\begin{aligned}
\left\langle\phi^{2}\right\rangle & =\frac{\ln (1-a / r)}{8 \pi^{2} r^{2}} \approx-\frac{a}{8 \pi^{2} r^{3}}-\frac{a^{2}}{16 \pi^{2} r^{4}} \\
\left\langle\dot{\phi}^{2}\right\rangle & =\frac{2 r-a}{32 \pi^{2}(r-a)^{2} r^{4}} \approx \frac{a}{16 \pi^{2} r^{5}}+\frac{3 a^{2}}{32 \pi^{2} r^{6}} \\
\left\langle\left(\partial_{r} \phi\right)^{2}\right\rangle & =\frac{5-6 r+4(r-a)^{2} \ln (1-a / r)}{32 \pi^{2}(r-a)^{2} r^{4}} \approx-\frac{5 a}{16 \pi^{2} r^{5}}-\frac{9 a^{2}}{32 \pi^{2} r^{6}} \\
\left\langle\left(\partial_{\perp} \phi\right)^{2}\right\rangle & =0
\end{aligned}
$$

where the approximate forms are those necessary to compute components to $O\left(r^{-6}\right)$.

Substituting these large $r$ approximations into Eqs. (7. .91$)$, we find the following stressenergy components

$$
\begin{aligned}
\rho & =-\frac{a}{8 \pi^{2} r^{5}}-\frac{3 a^{2}}{32 \pi^{2} r^{6}}+\xi\left[\frac{3 a}{4 \pi^{2} r^{5}}+\frac{3 a^{2}}{4 \pi^{2} r^{6}}\right] \\
p_{r} & =-\frac{a}{8 \pi^{2} r^{5}}-\frac{3 a^{2}}{32 \pi^{2} r^{6}}+\xi\left[\frac{3 a}{4 \pi^{2} r^{5}}+\frac{a^{2}}{2 \pi^{2} r^{6}}\right] \\
p_{\perp} & =\frac{3 a}{16 \pi^{2} r^{5}}+\frac{3 a^{2}}{16 \pi^{2} r^{6}}-\xi\left[\frac{9 a}{8 \pi^{2} r^{5}}+\frac{a^{2}}{\pi^{2} r^{6}}\right] .
\end{aligned}
$$


The leading terms agree with [10], with the correction that the fraction in Eq. (4.17) of [10] should be $(D-1) / D$ rather than $(D-2) /(D-1)$, where $D$ is the number of spatial dimensions [12].

For conformal coupling all terms of order $\left(1 / r^{5}\right)$ cancel to give

$$
\begin{array}{r}
\rho=\frac{a^{2}}{32 \pi^{2} r^{6}} \\
p_{r}=-\frac{a^{2}}{96 \pi^{2} r^{6}} \\
p_{\perp}=\frac{a^{2}}{48 \pi^{2} r^{6}} .
\end{array}
$$

Using Eqs. (35) 38) in Eq. (11) we find a large $r$ expression for the NEC,

$$
\begin{aligned}
T_{\mu \nu} V^{\mu} V^{\nu}= & \frac{a}{16 \pi^{2} r^{5}}+\frac{3 a^{2}}{32 \pi^{2} r^{6}}-v_{r}^{2}\left(\frac{5 a}{16 \pi^{2} r^{5}}+\frac{9 a^{2}}{32 \pi^{2} r^{6}}\right) \\
& +\xi\left[v_{r}^{2}\left(\frac{15 a}{8 \pi^{2} r^{5}}+\frac{3 a^{2}}{2 \pi^{2} r^{6}}\right)-\frac{3 a}{8 \pi^{2} r^{5}}-\frac{a^{2}}{4 \pi^{2} r^{6}}\right]
\end{aligned}
$$

For conformal coupling,

$$
T_{\mu \nu} V^{\mu} V^{\nu}=\frac{a^{2}}{32 \pi^{2} r^{6}}\left(\frac{5}{3}-v_{r}^{2}\right)>0
$$

so the NEC is obeyed far from the sphere.

\section{NUMERICAL RESULTS FOR THE NEC WITH CONFORMAL COUPLING}

We have computed the NEC contribution for the case of conformal coupling, $\xi=1 / 6$, by numerically performing the integral in equation Eq. (34). Fig. 1 shows the contributions from several partial waves for the case of radial motion. The case of tangential motion is very similar. The $\ell=0$ contribution is always positive, as shown in Eq. (46), but for other $\ell$, the sign depends on position. Thus, unlike the electromagnetic field case [13], the NEC does not hold in each channel individually.

Nevertheless, when we sum up all contributions, we find that the NEC is obeyed at any distance from the sphere, as shown in Fig. 2. Tangential motion yields a larger value for the NEC at any given point than radial, but both are always positive. Since the NEC is obeyed at each point, the ANEC is also obeyed.

The term depending on $\xi$ in the NEC, Eq. (10), is a total derivative in the direction $V$, so it gives no contribution to the ANEC integral in Eq. (2). Thus the ANEC does not depend on curvature coupling, and since it holds for conformal coupling it will hold for any coupling, as we found numerically in the minimally-coupled case [9].

\section{PERFECT MIRROR RESULT FROM SPHERICAL CASE}

In this section we will consider the leading-order behavior of the stress-energy tensor when we get very close to the sphere, the opposite limit to that of Sec. $\nabla$. In this case, there 


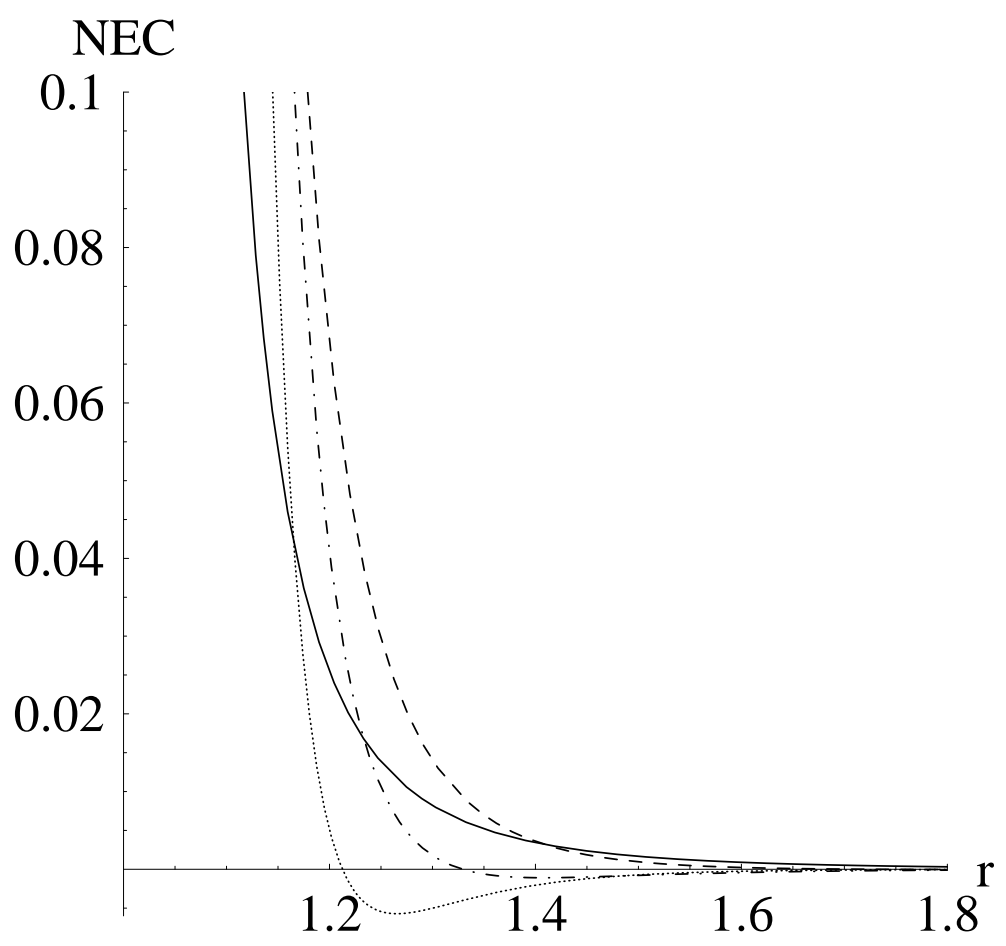

FIG. 1: We plot here the null energy condition, at a distance $r$ from the center of a sphere for motion in the radial direction for the $\ell=0$ (solid line), $\ell=2$ (dashed line), $\ell=4$ (dashed-dotted line), and $\ell=6$ (dotted line) partial waves.

will be a divergence due to summing over large numbers of partial wave modes, so we can use the large-order approximation for Bessel functions, as described in [10].

The expectation value of $\phi^{2}$ is given by Eq. (30), which we can write

$$
\left\langle\phi^{2}\right\rangle=-\sum_{\ell} \frac{\nu}{2 \pi^{2} r} \int_{0}^{\infty} d \kappa \frac{I_{\nu}(\kappa a)}{K_{\nu}(\kappa a)} K_{\nu}(\kappa r)^{2}
$$

where $\nu=\ell+1 / 2$.

Now we will consider the case where $\epsilon=r / a-1 \ll 1$. We let $x=\kappa a / \nu$ and $y=(1+\epsilon) x=$ $\kappa r / \nu$ to get

$$
\left\langle\phi^{2}\right\rangle=-\sum_{\ell} \frac{\nu^{2}}{2 \pi^{2} r a} \int_{0}^{\infty} d x \frac{I_{\nu}(\nu x)}{K_{\nu}(\nu x)} K_{\nu}(\nu y)^{2}
$$

Since we are summing over a large number of partial waves, we can use the large-order approximation to the Bessel functions. For $\nu \gg 1$ [14],

$$
\begin{aligned}
I_{\nu}(\nu x) & \approx \frac{1}{\sqrt{2 \pi \nu}} \frac{e^{\nu \eta}}{\left(1+x^{2}\right)^{1 / 4}} \\
K_{\nu}(\nu x) & \approx \sqrt{\frac{\pi}{2 \nu}} \frac{e^{-\nu \eta}}{\left(1+x^{2}\right)^{1 / 4}}
\end{aligned}
$$




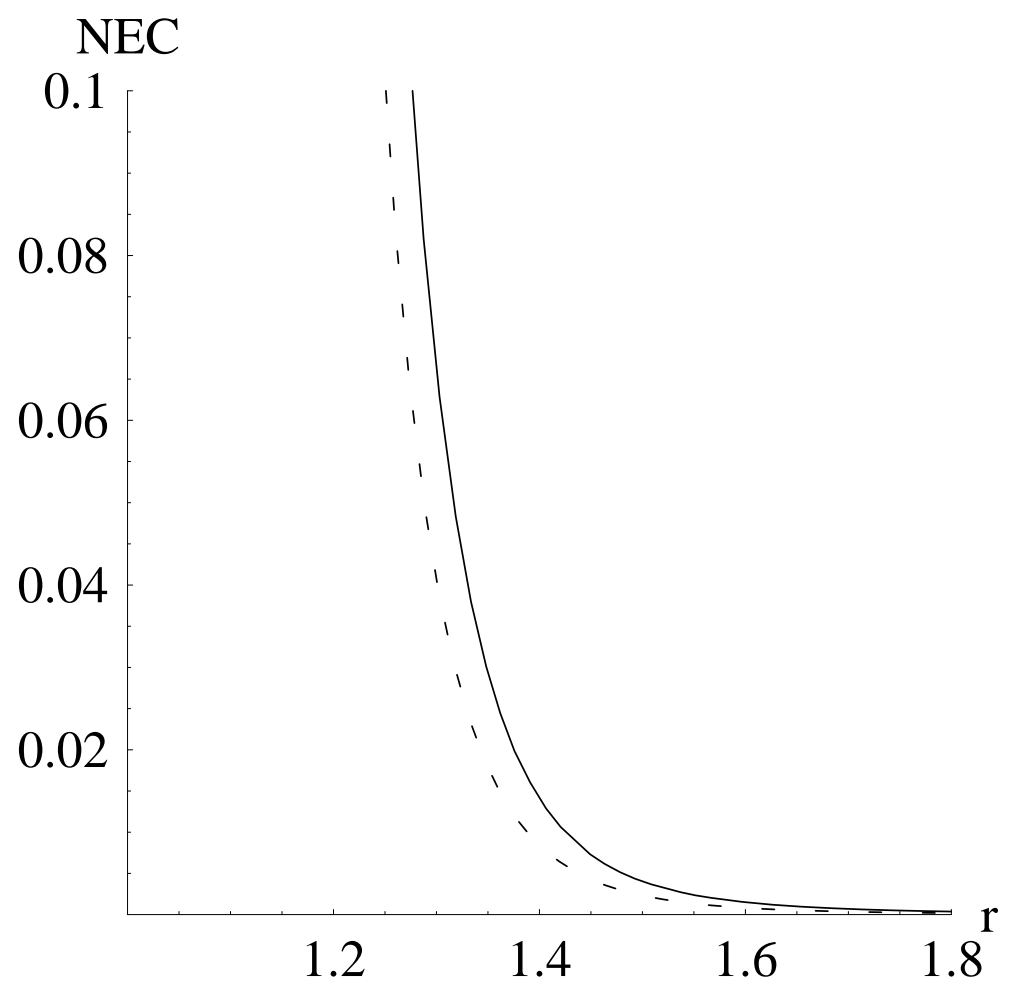

FIG. 2: The null energy condition at a distance $r$ from the center of a sphere for motion in the azimuthal (solid line) and radial (dashed line) directions.

where

$$
\eta(x)=\sqrt{1+x^{2}}+\ln \frac{x}{1+\sqrt{1+x^{2}}}
$$

So

$$
\left\langle\phi^{2}\right\rangle \approx-\frac{1}{4 \pi^{2} a^{2}} \sum_{\ell} \nu \int_{0}^{\infty} \frac{d x}{\sqrt{1+x^{2}}} e^{-2 \nu(\eta(y)-\eta(x))}
$$

We have ignored higher order terms in $\epsilon$ by replacing $r$ with $a$ in the prefactor and $y$ with $x$ in the square root.

We expand to first order,

$$
\eta(y)-\eta(x)=\epsilon x \frac{d \eta}{d x}=\epsilon \sqrt{1+x^{2}}
$$

so we have

$$
\int_{0}^{\infty} \frac{d x}{\sqrt{1+x^{2}}} e^{-2 \nu \epsilon \sqrt{1+x^{2}}}=K_{0}(2 \nu \epsilon)
$$

Thus

$$
\left\langle\phi^{2}\right\rangle \approx-\sum_{\ell} \frac{\nu}{4 \pi^{2} a^{2}} K_{0}(2 \nu \epsilon)
$$

We approximate the sum by an integral,

$$
\left\langle\phi^{2}\right\rangle \approx-\int_{0}^{\infty} d \nu \frac{\nu}{4 \pi^{2} a^{2}} K_{0}(2 \nu \epsilon)=-\frac{1}{16 \pi^{2} a^{2} \epsilon^{2}}=-\frac{1}{16 \pi^{2}(r-a)^{2}} .
$$


From Eq. (31), the time derivative term differs from $\left\langle\phi^{2}\right\rangle$ by a factor $-\kappa^{2}$ in the integrand. By the analysis above,

$$
\left\langle\dot{\phi}^{2}\right\rangle \approx \sum_{\ell} \frac{\nu^{3}}{4 \pi^{2} a^{4}} \int_{0}^{\infty} d x \frac{x^{2}}{\sqrt{1+x^{2}}} e^{-2 \nu(\eta(y)-\eta(x))}
$$

Using

$$
\int_{0}^{\infty} d x \frac{x^{2}}{\sqrt{1+x^{2}}} e^{-2 \nu \epsilon \sqrt{1+x^{2}}}=\frac{K_{1}(2 \nu \epsilon)}{2 \nu \epsilon}
$$

we find

$$
\left\langle\dot{\phi}^{2}\right\rangle \approx \sum_{\ell} \frac{\nu^{2}}{8 \pi^{2} a^{4} \epsilon} K_{1}(2 \nu \epsilon) \approx \int_{0}^{\infty} d \nu \frac{\nu^{2}}{8 \pi^{2} a^{4} \epsilon} K_{1}(2 \nu \epsilon)=\frac{1}{32 \pi^{2} a^{4} \epsilon^{4}}=\frac{1}{32 \pi^{2}(r-a)^{4}}
$$

Since $K_{1}(z) \sim 1 / z$ for small $z$, the contribution from a single partial wave mode with $\ell \ll 1 / \epsilon$ is proportional to $\ell / \epsilon^{2}$. The sum over these modes thus diverges as $\ell^{2}$. It is truncated when $\ell \sim 1 / \epsilon$, so the final result is proportional to $1 / \epsilon^{4}$.

From Eq. (33), the tangential term differs from $\left\langle\phi^{2}\right\rangle$ by a factor $\ell(\ell+1) /\left(2 r^{2}\right) \approx \nu^{2} /\left(2 a^{2}\right)$ since we are keeping only the highest power of $\nu$ and lowest order in $\epsilon$. Thus we find

$$
\left\langle\left(\partial_{\perp} \phi\right)^{2}\right\rangle \approx-\int_{0}^{\infty} d \nu \frac{\nu^{3}}{8 \pi^{2} a^{4}} K_{0}(2 \nu \epsilon)=-\frac{1}{32 \pi^{2} a^{4} \epsilon^{4}}=-\frac{1}{32 \pi^{2}(r-a)^{4}}
$$

The angular derivative brings in a factor of $\ell$, so this term has two more powers of $\ell$ and correspondingly two fewer powers of $\epsilon$ in the denominator. For $\ell \ll 1 / \epsilon$ it goes as $\ell^{3} \ln \nu \epsilon$, which is much smaller than the time-derivative term, even though the sum over $\ell$ is the same except for sign.

From Eq. (32), the radial term differs from $\left\langle\dot{\phi}^{2}\right\rangle$ by a factor $-k_{\ell}^{\prime}(\kappa r)^{2} / k_{\ell}(\kappa r)$ in the integrand. The derivative of the spherical Bessel function is

$$
k_{\ell}^{\prime}(z)=-\frac{\ell}{2 \ell+1} k_{\ell+1}(z)-\frac{\ell+1}{2 \ell+1} k_{\ell-1}(z)
$$

while the derivative of the regular Bessel function is

$$
K_{\ell}^{\prime}(z)=-\frac{1}{2} K_{\ell+1}(z)-\frac{1}{2} K_{\ell-1}(z)
$$

but the difference in the coefficients does not matter for $\ell$ large. Thus

$$
\frac{k_{\ell}^{\prime}(z)}{k_{\ell}(z)} \approx \frac{K_{\ell}^{\prime}(z)}{K_{\ell}(z)}
$$

The derivative for large order is

$$
K_{\nu}^{\prime}(\nu y) \approx-\sqrt{\frac{\pi}{2 \nu}} \frac{\left(1+y^{2}\right)^{1 / 4}}{y} e^{-\nu \eta}=-\frac{\sqrt{1+y^{2}}}{y} K_{\nu}(\nu y)
$$

Thus we find

$$
\frac{k_{\ell}^{\prime}(\kappa r)^{2}}{k_{\ell}(\kappa r)^{2}} \approx 1+\frac{1}{y^{2}}=1+\frac{\nu^{2}}{\kappa^{2} r^{2}}
$$


Consequently

$$
\left\langle\left(\partial_{r} \phi\right)^{2}\right\rangle \approx 2\left\langle\left(\partial_{\perp} \phi\right)^{2}\right\rangle-\left\langle\dot{\phi}^{2}\right\rangle \approx-\frac{3}{32 \pi^{2}(r-a)^{4}}
$$

For a conformal field we will also need

$$
\partial_{r}^{2}\left\langle\phi^{2}\right\rangle=-\frac{3}{8 \pi^{2}(r-a)^{4}}
$$

from Eq. (56), but we will not need $\partial_{r}\left\langle\phi^{2}\right\rangle$ because that does not diverge as $(r-a)^{-4}$.

Putting everything together, the stress tensor components to order $1 /(r-a)^{4}$ are

$$
\begin{aligned}
\rho & \approx \frac{1}{2}\left[\dot{\phi}^{2}+\left(\partial_{r} \phi\right)^{2}+2\left(\partial_{\perp} \phi\right)^{2}\right]-\xi \partial_{r}^{2}\left(\phi^{2}\right) \approx-\frac{1-6 \xi}{16 \pi^{2}(r-a)^{4}} \\
p_{r} & \approx \frac{1}{2}\left[\dot{\phi}^{2}+\left(\partial_{r} \phi\right)^{2}-2\left(\partial_{\perp} \phi\right)^{2}\right] \approx 0 \\
p_{\perp} & \approx \frac{1}{2}\left[\dot{\phi}^{2}-\left(\partial_{r} \phi\right)^{2}\right]+\xi \partial_{r}^{2}\left(\phi^{2}\right) \approx \frac{1-6 \xi}{16 \pi^{2}(r-a)^{4}}
\end{aligned}
$$

For minimal coupling we get the standard result for a single, flat Dirichlet plate, and for conformal coupling, $\xi=1 / 6$, we also get the standard vanishing result for the flat plate. Therefore, by linearity, the result is correct for general coupling.

\section{CONCLUSIONS}

We have studied the problem of a real, massless scalar field outside a reflecting sphere, in the calculational framework developed in [11]. We obtained expressions for the stressenergy tensor for general curvature coupling in agreement with [10]. We analyzed the null energy condition for the specific case of conformal coupling and showed, analytically for large distances, and numerically everywhere, that it is obeyed.

This implies that the averaged null energy condition is obeyed for conformal coupling in this system. Since the ANEC is independent of the curvature coupling, it is obeyed for any coupling, in accord with our conjecture in [9] that the ANEC is satisfied for all geodesics which pass outside any localized background potential.

We have also given a derivation of the standard stress-energy components for a flat Dirichlet plate as the close-in limit of the spherical geometry considered here. The wellknown divergence as one approaches the surface arises in this case from a summation over many angular momentum modes.

\section{ACKNOWLEDGMENTS}

We would like to thank Noah Graham and Aram Saharian for helpful conversations. K. D. O. was supported in part by the National Science Foundation.

[1] H. B. G. Casimir, Proc. Kon. Ned. Akad. Wet. B51, 793 (1948).

[2] S. K. Lamoreaux, Phys. Rev. Lett. 78, 5 (1997). 
[3] M. S. Morris, K. S. Thorne, and U. Yurtsever, Phys. Rev. Lett. 61, 1446 (1988).

[4] K. D. Olum, Phys. Rev. Lett. 81, 3567 (1998), gr-qc/9805003.

[5] S. W. Hawking, Phys. Rev. D 46, 603 (1992).

[6] G. J. Galloway, Manuscr. Math. 35, 209 (1981).

[7] T. A. Roman, Phys. Rev. D 33, 3526 (1986).

[8] T. A. Roman, Phys. Rev. D 37, 546 (1988).

[9] D. Schwartz-Perlov and K. D. Olum, Phys. Rev. D68, 065016 (2003), hep-th/0307067.

[10] A. A. Saharian, Phys. Rev. D63, 125007 (2001), hep-th/0012185.

[11] N. Graham and K. D. Olum, Phys. Rev. D67, 085014 (2003), hep-th/0211244.

[12] A. A. Saharian, personal communication.

[13] N. Graham, K. D. Olum, and D. Schwartz-Perlov, Phys. Rev. D70, 105019 (2004), hepth/0407006.

[14] M. Abramowitz and I. A. Stegun, Handbook of Mathematical Functions With Formulas, Graphs, and Mathematical Tables (U.S. government printing office, Washington, 1972). 